\title{
Serological Response of Swine to an Attenuated Salmonella enterica serovar Typhimurium Strain that Reduces Gastrointestinal Colonization, Fecal Shedding and Disease due to Virulent Salmonella Typhimurium
}

\author{
Bearson, B.L. * ${ }^{*(1)}$ \\ Bearson, S.M.D.(2) \\ "I'National Laboratory for Agriculture and the Environment, ARS, USDA, Ames, IA, USA and \\ ${ }^{(2)}$ National Animal Disease Center, ARS, USDA, Ames, IA, USA \\ *2110 University Drive, NSRIC-2 103, 50011 , Ames (IA), USA \\ e-mail: brad.bearson@ars.usda.gov; fax: 15152941209
}

\begin{abstract}
Swine are often asymptomatic carriers of Salmonella spp. Interventions are needed to limit Salmonella colonization of swine to enhance food safety. An attenuated Salmonella enterica serovar Typhimurium mutant strain (BBS 202) was tested in swine to determine whether vaccination could provide protection against wild-type $S$. Typhimurium challenge. Two groups of piglets ( $n=14 /$ group) received an intranasal inoculation of BBS 202 or a PBS placebo at 6-weeks of age with a booster 2-weeks later. At 11 -weeks of age, all pigs were challenged with the parental, wild-type S. Typhimurium by intranasal inoculation. Average swine rectal temperature (fever) was significantly decreased in BBS 202-vaccinated pigs at days 1 and 2 post-challenge with virulent $S$. Typhimurium compared to mock-vaccinated pigs. Fecal shedding of wild-type S. Typhimurium was significantly reduced at 2-days post-challenge in BBS 202-vaccinated pigs compared to mock-vaccinated pigs. Colonization of tissues within the gastrointestinal tract by wild-type $S$. Typhimurium was reduced in BBS 202-vaccinated pigs; a significant decrease in S. Typhimurium colonization of the ileal Peyer's patch region and ileocecal lymph nodes at 7-days post-challenge was observed. Serological analysis using the IDEXX HerdChek Swine Salmonella Test Kit indicated that all pigs were negative for antibodies to LPS derived from Salmonella serogroups B, C1, and D prior to challenge with wild-type S. Typhimurium. Thus, although vaccinated pigs had received two doses of BBS 202, antibodies from these pigs were not reactive to the LPS antigen in the ELISA test. However, sera from $85 \%$ of vaccinated and $78 \%$ of mock-vaccinated pigs were positive in the ELISA assay at day-7 post-challenge with wild-type S. Typhimurium. These preliminary results indicate that vaccination of swine with BBS 202 confers protection against challenge with virulent S. Typhimurium by reducing disease severity, pathogen fecal shedding, and gastrointestinal colonization but does not interfere with herd level monitoring for Salmonella spp., thereby allowing for differentiation between pigs naturally infected with Salmonella spp. and BBS 202-vaccinated swine.
\end{abstract}

\section{Introduction}

An estimated 1 million cases of foodborne illness are attributed to nontyphoidal Salmonella spp. each year in the U.S. at a predicted cost of \$2.3 billion (Scallan, 2011 ; Frenzen, 1999). Nontyphoidal Salmonella spp. are a leading cause of hospitalization (35\%) and death (28\%), in the U.S., due to foodborne disease (Scallan, 2011 ). There are greater than 2,400 Salmonella serovars that are ubiquitous in the environment and many can colonize food producing animals and poultry as well as wild animals and birds without causing overt disease. In the U.S., 100,000 cases of pork-associated, human salmonellosis occur each year with a social cost of \$81 million annually (Miller, 2005). Swine 2006, the most recent study from the USDA's National Animal Health Monitoring System, reported that 52.6\% of swine production sites (representing $94 \%$ of the U.S. swine inventory) were positive for Salmonella spp. Swine that are Salmonella carriers are a food safety risk for consumers of pork, an animal health risk to non-colonized/uninfected pigs, and an environmental risk due to fecal shedding of the pathogen into swine manure that is used as a soil amendment. Interventions are needed to limit the colonization of swine with Salmonella spp. and reduce the risks to public health, animal health and the environment. We investigated an attenuated Salmonella enterica serovar Typhimurium strain to determine whether administration of the potential vaccine strain would 1/ protect swine against virulent S. Typhimurium challenge while 2) allowing the differentiation of infected and vaccinated animals (DIVA) using the IDEXX HerdChek Swine Salmonella Test Kit. Our results indicate that BBS 202-vaccinated pigs have reduced disease severity, pathogen fecal shedding, and gastrointestinal colonization due to virulent $\mathrm{S}$. Typhimurium challenge, but swine vaccination did not interfere with the herd level monitoring system for Salmonella spp. 


\section{Material and Methods}

The wild-type S. Typhimurium strain $\chi 4232$ and its attenuated derivative BBS 202 were used in this study. At 6-weeks of age, two groups of piglets ( $n=14$ /group), fecal-negative for Salmonella spp., received an intranasal inoculation of $1 \mathrm{ml}$ PBS containing 1.0 X 109 CFU of BBS 202 or a PBS placebo. A booster of BBS 202 or PBS was given to the same groups of pigs 2-weeks later. At 11 -weeks of age, all pigs were challenged with $1 \mathrm{ml}$ PBS containing $1.0 \times 108 \mathrm{CFU}$ of $\chi 4232$ by intranasal inoculation. On $0,1,2,3$, and 7 days postinoculation (dpi), the swine rectal temperature was monitored, fecal samples were obtained for quantitative and qualitative Salmonella culture analysis, and blood samples were obtained via the jugular vein. At $7 \mathrm{dpi}$, all swine were euthanized and necropsies performed to obtain tissue samples from the gastrointestinal tract (ileal Peyer's Patch, ileocecal lymph nodes, and cecum) for quantitative and qualitative Salmonella culture analysis. Serum antibody analysis to LPS antigen derived from Salmonella serogroups B, C1, and D was performed using the IDEXX HerdChek Swine Salmonella Test Kit. Statistical analysis was performed with SAS Analyst (Cary, NC) using the Two Sample t-test for the Means. Results were considered statistically significant when P 0.05.

\section{Results}

A schematic for swine vaccination and challenge is shown below.

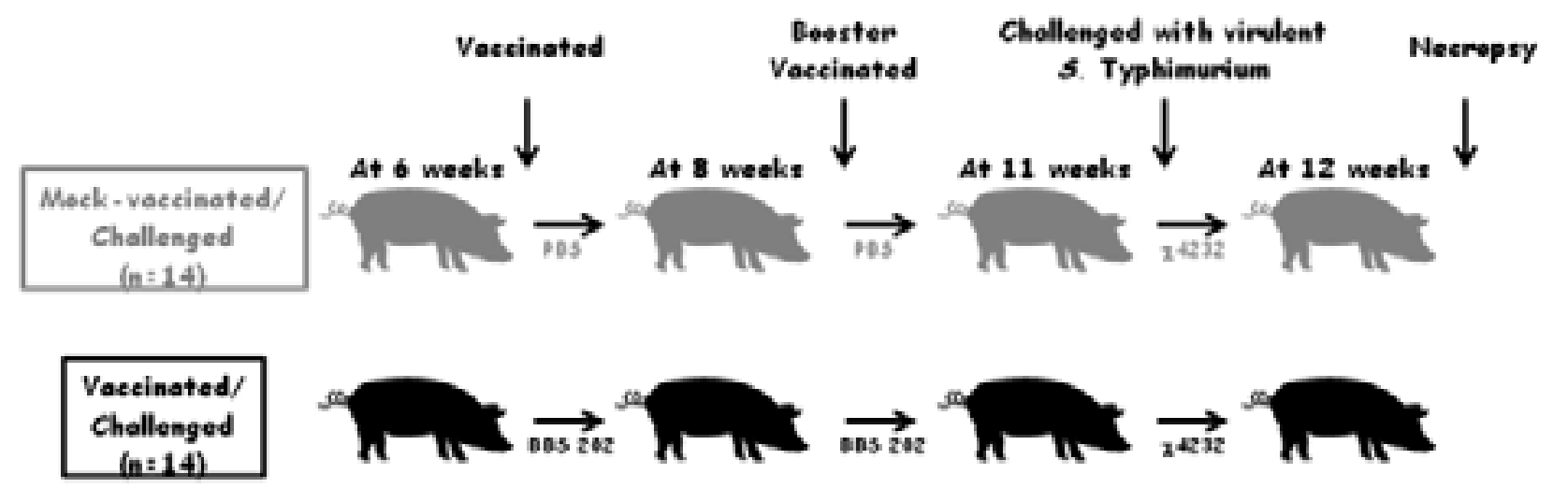

At 1 and 2 dpi, the swine rectal temperature was significantly increased in mock-vaccinated pigs compared to BBS 202-vaccinated pigs (Figure 1).

Figure 1. The average rectal temperature of BBS 202-vaccinated pigs is significantly lower at 1 and 2 days post-inoculation with wild-type $\mathrm{S}$. Typhimurium compared to mock-vaccinated pigs. The swine rectal temperature was monitored on $\mathrm{O}$, $1,2,3$, and 7 days post-inoculation. ${ }^{*}$ Significant difference comparing BBS 202-vaccinated to mock-vaccinated pigs.

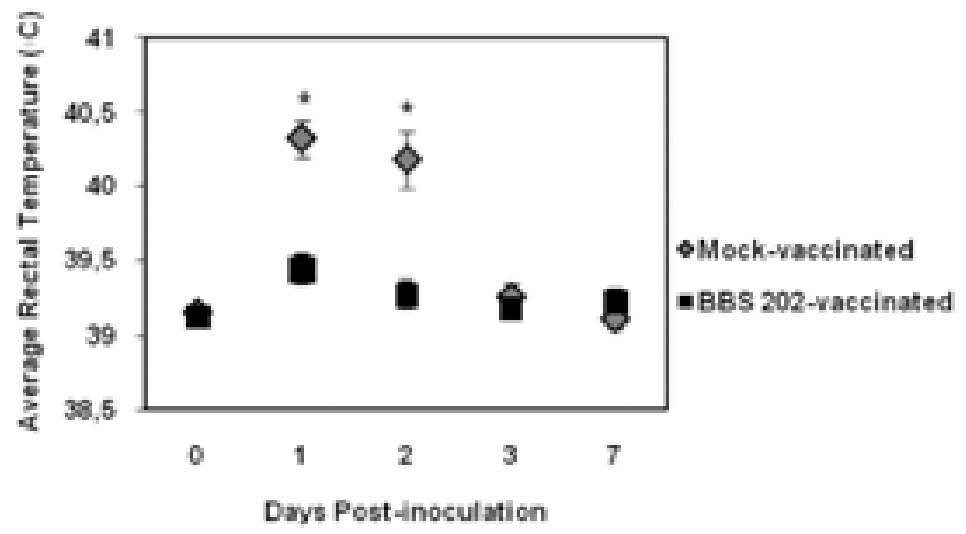

Swine fecal shedding of $\mathrm{S}$. Typhimurium was significantly increased at day 2 post-challenge in mock-vaccinated pigs compared to BBS 202-vaccinated pigs (Figure 2). 
Figure 2. Swine fecal shedding of wild-type $\mathrm{S}$. Typhimurium is significantly lower at 2 days postinoculation in BBS 202-vaccinated pigs compared to mock-vaccinated pigs. Swine fecal samples were obtained on 1, 2, 3, and 7 days postinoculation for quantitative and qualitative Salmonella bacteriological analysis. *Significant difference comparing BBS 202-vaccinated to mock-vaccinated pigs at the indicated time point.

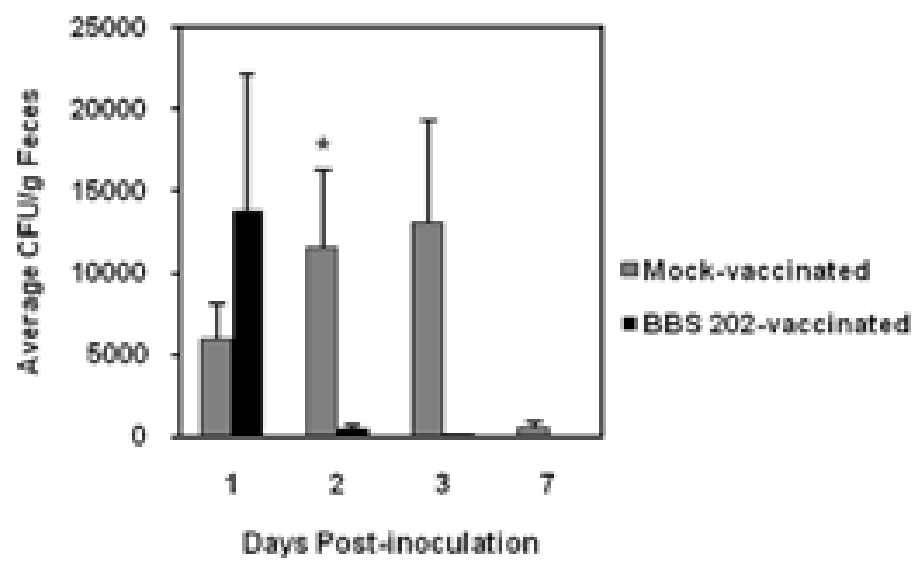

S. Typhimurium colonization of the ileocecal lymph nodes and ileal Peyer's Patch at 7 days post-challenge was significantly decreased in BBS 202-vaccinated pigs compared to mock-vaccinated pigs (Figure 3).

Figure 3. Colonization of the lleocecal Lymph Nodes and Peyer's Patches by wild-type S. Typhimurium is significantly lower at 7 days post-inoculation in BBS 202-vaccinated pigs compared to mock-vaccinated pigs. At 7 days post-inoculation, all pigs were necropsied and tissue samples of the gastrointestinal tract [ileocecal lymph nodes (ICLN), Peyer's Patch (PP), cecum] were obtained for quantitative and qualitative Salmonella bacteriological analysis. *Significant difference comparing BBS 202-vaccinated to mock-vaccinated pigs for the indicated tissue.

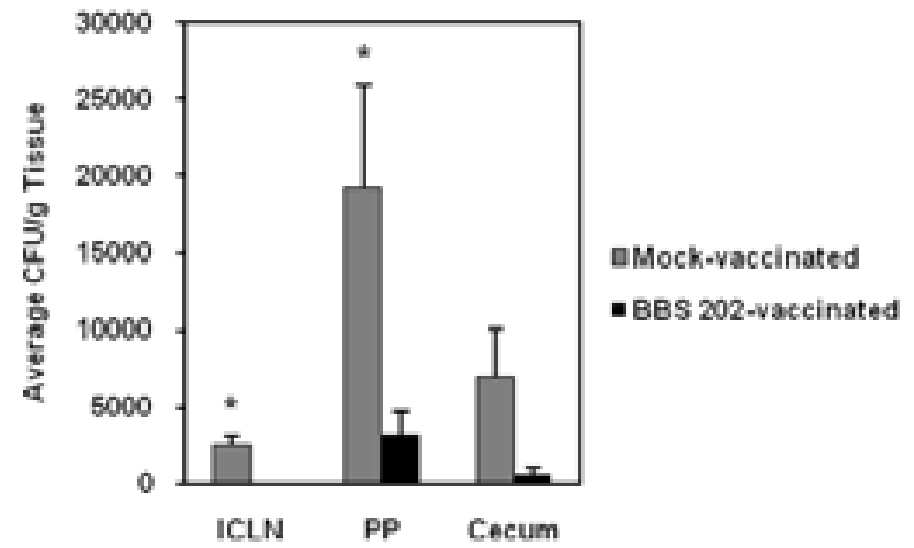

Swine serum was obtained from all 28 pigs at 11 -weeks of age 15 weeks following initial vaccination with BBS 202 and the day of challenge) to determine the presence or absence of antibodies to Salmonella LPS antigen using the IDEXX HerdChek Swine Salmonella Test Kit; all swine were negative to LPS antigen by ELISA (Figure 4). At 12-weeks of age (7-days post challenge), swine serum was obtained to determine seroconversion to Salmonella LPS antigen following wild-type challenge. Sera from $85 \%$ of vaccinated and $78 \%$ of mock-vaccinated pigs were positive in the LPS ELISA assay at 12 -weeks of age (7 dpi).

Figure 4. Serum from BBS 202-vaccinated swine is negative in the IDEXX HerdChek Swine Salmonella Test Kit 5-weeks after initial vaccination. Swine serum from all pigs at 1 1- and 12-weeks of age was assayed for Salmonella LPS antigen using the IDEXX test. The percent positive pigs at each time point are shown. ${ }^{*}$ All pigs were challenged with wild-type $S$. Typhimurium at 11 -weeks of age. 


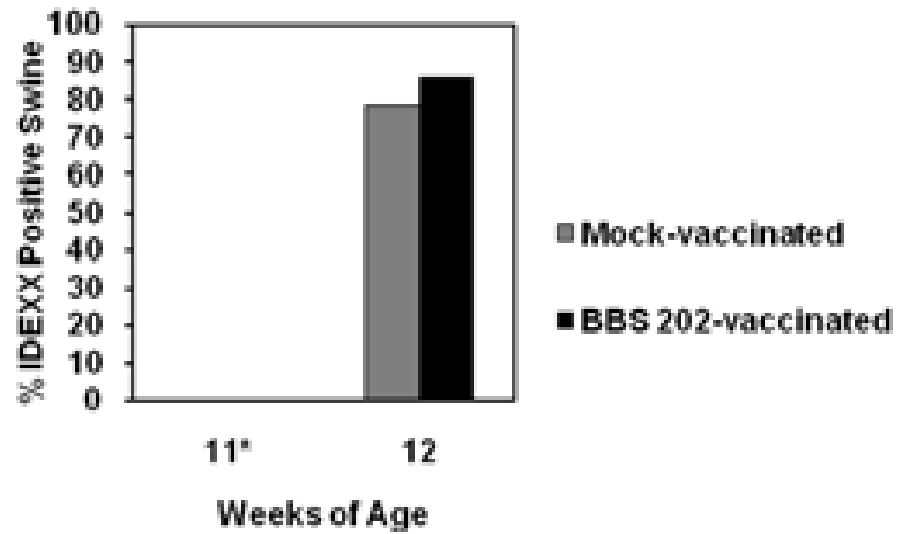

\section{Discussion}

Salmonella spp. can colonize the gastrointestinal tract of pigs without causing obvious disease. Swine that are Salmonella carriers pose a risk to public health, animal health and the environment. Vaccination of swine against Salmonella spp. may reduce pathogen colonization and carriage but may also interfere with swine herd Salmonella surveillance programs due to production of anti-Salmonella LPS antibodies. Our rationally attenuated S. Typhimurium vaccine strain was designed to overcome this potential vaccine limitation and allow the differentiation of infected and vaccinated swine. Indeed, vaccination with BBS 202 reduced disease severity, Salmonella fecal shedding and gastrointestinal colonization following challenge with virulent $S$. Typhimurium, without stimulating an immune response to $S$. Typhimurium LPS antigens. Furthermore, upon challenge with a virulent, wild-type S. Typhimurium strain, the majority of both BBS 202-vaccinated and mock-vaccinated swine had a seroconversion to a positive IDEXX ELISA reaction indicating a recent Salmonella infection.

\section{Conclusion}

Vaccination of swine with the S. Typhimurium BBS 202 vaccine strain reduces S. Typhimurium gastrointestinal colonization and fecal shedding but did not stimulate an anti-Salmonella LPS immune response that would compromise Salmonella surveillance programs for swine herds. Thus, vaccination with the S. Typhimurium BBS 202 vaccine strain permits the differentiation of Salmonella infected versus vaccinated swine.

\section{References}

Frenzen P., et. al., 1999, Salmonella Cost Estimate Updated Using FoodNet Data, FoodReview, 22: 1 0-15. Miller G.Y., et. al., 2005, In囚uence of Salmonella in Pigs Preharvest and during Pork Processing on Human Health Costs and Risks from Pork, Int. Ass. Food Prot., J. Food Prot., 68:1788-1798.

NAHMS. 2009. Salmonella on U.S. Swine Sites - Prevalence and Antimicrobial Susceptibility. http://www.aphis.usda. gov/animal_health/nahms/swine/index.shtml\#swine2006.

Scallan E., et. al., 201 1, Foodborne Illness Acquired in the United States-Major Pathogens, CDC, Emerg. Infect. Dis., 17:7-15. 